\title{
Is it possible to predict the side of hepatic metastases according to the primary location of colorectal cancer?
}

\author{
Ayşegül Gürsoy Çoruh ${ }^{1 A, D, E}$, Çağlar Uzun ${ }^{1 A, D}$, Esra Bozca ${ }^{2 B}$, Büşra Bozca ${ }^{2 B}$, İhsan Batuhan Demir ${ }^{2 B}$, \\ Hilal Gülseren Atasever ${ }^{2 B}$, Berna Göçtürk ${ }^{2 B}$, Batuhan Bakırarar ${ }^{3 C}$, Cihangir Akyol ${ }^{2 D}$ \\ 'Department of Radiology, School of Medicine, Ankara University, Ankara, Turkey \\ 2Department of General Surgery, School of Medicine, Ankara University, Ankara, Turkey \\ ${ }^{3}$ Department of Biostatistics, School of Medicine, Ankara University, Ankara, Turkey
}

\section{Abstract}

Purpose: The aim of this study was to investigate the impact of the primary location of colorectal adenocarcinoma on the lobar distribution of its hepatic metastases based on the streamline hypothesis.

Material and methods: The hospital database was utilised to identify the colorectal cancer patients. Eighty-six patients diagnosed with colorectal adenocarcinoma, who had hepatic metastases on the initial diagnostic stage or on the follow-up investigations, were enrolled the study. Computed tomography (CT) images of the study population were reviewed for the primary location of the colorectal tumour, and the side and number of hepatic metastases.

Results: A total of 481 metastases were counted on CT from 22 right-sided and 64 left-sided colon tumours. The ratio of right-to-left hemiliver involvement was $1.97: 1$ for whole study population. The right-to-left ratio was calculated as 1.55 $: 1$ for right colon tumours and $2.17: 1$ for left colon tumours $(p=0.106)$. In the subgroup analysis with unilobar metastatic patients, again there was no significant difference in terms of the colorectal tumours' primary location $(p=0.325)$. Conclusions: The lobar distribution of hepatic metastases from colorectal adenocarcinoma may not be associated with the primary tumour localisation

Key words: colorectal neoplasms, metastasis, liver, portal vein, streamline phenomenon.

\section{Introduction}

Colorectal carcinoma is the third most common cancer worldwide and one of the most common causes of cancer-related deaths, with high mortality rates especially in the advanced stage of the disease [1,2]. Colorectal carcinoma represents two different spectra of disease in the same organ according to the location (right/left colon) of the primary tumour [3]. It acts distinctively in terms of metastatic spread, disease progression, and survival, according to the primary tumour location. Those different behaviours may be due to arising from different embryologic divisions (midgut/hindgut). In addition, the genetic and epigenetic features of the tumour also differ according to the tumour localisation $[3,4]$.

The stage of the disease and the presence of metastasis have a significant effect on survival. The five-year survival rate is approximately $90 \%$ for early-stage disease. However, it decreases to $10 \%$ in advanced-stage patients with distant metastasis [5]. The liver is one of the most common sites of metastasis due to portal venous circulation [6]. Two-thirds of liver blood flow derives from portal circulation [7]. The superior mesenteric vein (SMV), splenic vein, and inferior mesenteric vein (IMV), which drains into the splenic vein or confluence, form the portal vein. The cecum, ascending colon, and transverse colon are drained by the

Correspondence address:

Cihangir Akyol, Department of General Surgery, School of Medicine, Ankara University, Ankara, Turkey, Talatpaşa Bulvarı, Sihhiye, 06100 Ankara, Turkey,

e-mail: cihangirakyol@gmail.com 
SMV; however, the veins from the descending colon, sigmoid colon, and upper rectum drain into the IMV. Human and animal studies have demonstrated a partial partition of blood flow (which is called streamline flow) in the portal vein $[8,9]$. Moore et al. showed by using portal venography that the left lobe receives a large amount of blood from the splenic vein, whereas the right lobe receives the greatest amount of blood from the SMV [9]. Therefore, the aim of this study is to investigate the impact of the primary tumour location on the lobar distribution of the liver metastasis from colorectal cancers according to the streamline flow of the portal vein.

\section{Material and methods}

\section{Study population}

The study protocol of this retrospective study was approved by the Institutional Review Board (ref no: I3-10619). The institution's Radiology Information System/Picture Archiving and Communication System (RIS/PACS; Centricity 5.0 RIS-i, GE Healthcare, Milwaukee, WI, USA) was used to identify patients with colorectal adenocarcinoma. Following the identification of colorectal adenocarcinoma patients who were diagnosed between November 2012 and April 2019 and were pathologically proven, computed tomography (CT) images and pathology reports were reviewed for the presence of hepatic synchronous or metachronous metastases. Patients with other distant metastases $(n=80)$ accompanied with liver metastasis (lung, bone, brain), patients who had synchronous and metachronous colorectal tumours $(n=5)$, patients who were diagnosed with low-mid rectal adenocarcinoma $(n=17$; lower rectal tumours have different venous flows, which drain into systemic veins), and patients who had motion artifacts on CT $(n=8)$ were excluded from the study. Patients with primary liver metastasis from colorectal adenocarcinoma who had undergone surgical resection at diagnosis and patients who developed only liver metastasis (Figure 1) on follow-up imaging investigations were enrolled in the study. Only the first surgery and first recurrence CT imaging study with hepatic metastasis were included in the study for patients with multiple recurrences. In total, 86 patients were included in the study. Out of 86 patients, two were diagnosed with mucinous adenocarcinoma. At our institution, patients were followed-up with laboratory tests and CT every six months for up to three years and subsequently if no signs of recurrence observed, patients followed by annually intervals. Figure 2 represents the flow chart of the study population.

\section{Computed tomography imaging and analyses}

CT examinations were performed on a 64-slice CT (Toshiba Aquilion 64, Japan) and a 16-slice CT (Siemens Somatom Sensation 16, Forcheim, Germany). At 2.5 hours prior to the study, patients ingested a mixture of $1500 \mathrm{ml}$ water with $50 \mathrm{ml}$ Urografin. In the current study, 60-100 ml (1-1.5 ml/kg) of intravenous contrast agent (350/100 Omnipaque, GE healthcare, Oslo, Norway) was administered at a rate of $2.5 \mathrm{ml} / \mathrm{s}$ via the antecubital vein. CT images were acquired using the following parameters: $120 \mathrm{kVp}$, collimations of $16 \times 1.2$ and $64 \times 0.5$, axial reconstructions with 1.2 and $1 \mathrm{~mm}$ slice thickness, and $1 \mathrm{~mm}$ slice intervals. CT examinations were performed by using a single phase (portal venous phase) or multiphase (arterial, portal venous, and delayed phase).

Of the 86 patients, 52 underwent surgery, and metastasis was confirmed by pathology. Twenty-one patients proved to have a hepatic metastasis via fine-needle aspiration biopsy (FNAB). In 13 patients with pathologically unproven and 21 patients with FNAB whose metastases were not pathologically proven, hepatic metastasis confirmation was done by two radiologists in consensus (AGC-ÇU) via follow-up screening (CT) examinations. After the confirmation of metastasis, the hepatic lesions were counted in the CT by the examiners (AGC-ÇU).
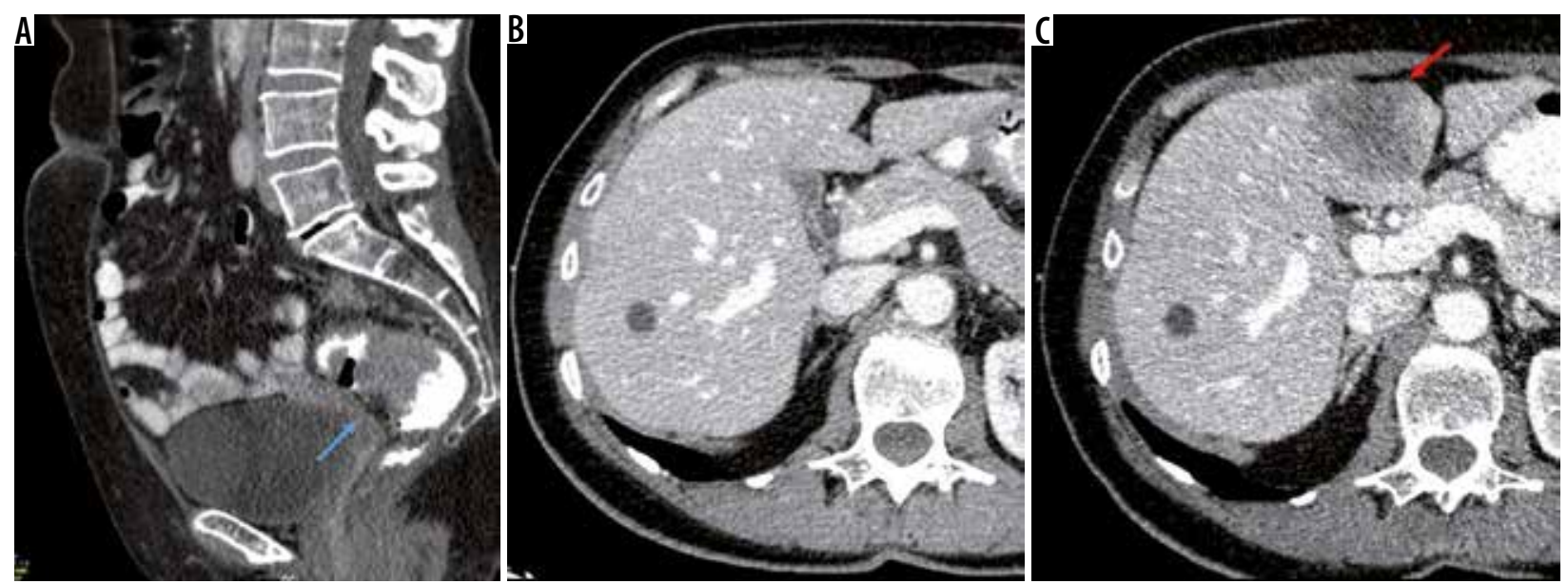

Figure 1. A) Sagittal enhanced computed tomography (CT) images of a 56-year-old female patient diagnosed with upper rectum (blue arrow) adenocarcinoma (T3N1M0). B) At diagnosis). C) Follow-up (T was performed 12 months after diagnosis, and a hypodense metastatic mass was observed on segment 4b on axial enhanced CT images (red arrow) 
The axial and multiplanar reformatted CT images of patients were evaluated for the counting of hepatic metastases and primary tumour location by the examiners, who had more than 10 years (AGC) and 18 years (ÇU) of experience in gastrointestinal imaging, on a workstation (Advantage Workstation 4.3; GE Healthcare, Waukesha, WI, USA). The segmental location of liver metastasis was concluded according to Couinaud's description. The right and left hemiliver distinction was performed by using the Cantlie line. With reference to this, the left hemiliver consists of segments I, II, III, and IV, and the right hemiliver consists of segments V, VI, VII, and VIII. The patients with tumours located between the transverse colon and cecum were referred to as the right colon tumour group, while the patients who had tumours between the splenic flexure and upper rectum constituted the left colon tumour group.

\section{Statistical analysis}

All statistical analyses were performed using the SPSS for Windows 11.5 software program (SPSS Inc., Chicago, IL). For the quantitative variables, mean \pm standard deviation and median (minimum-maximum) were used, and for the categorical variables, numbers (percentage) were used as descriptors in the study. The $\chi^{2}$ test was used to examine the relationship between the two categorical variables. The significance level was set at $p=0.05$.

\section{Results}

The study population consisted of 63 males (73.3\%) and 23 females $(26.7 \%)$ with a mean age of $66.75 \pm 11.04$ years
Colorectal adenocancer patients between November 2012 and April 2019

Exclusion criteria $(n=120)$

- Patients who had synchronous and metachronous colorectal tumors $(n=5)$

- Patients with other synchronous distant (lung, bone, brain) metastases $(n=80)$

- Patients with low-mid rectal adenocarcinoma $(n=17)$

- Patients with motion artifacts on computed tomography $(n=8)$

Inclusion criteria $(n=86)$

- Colorectal adenocancer (pathologically proven) patients with synchronous and metachronous liver metastases

- Only the first surgery and first recurrence imaging study (computed

tomography) with hepatic metastases

Figure 2. Flowchart of the study population

(range 35-92). Right colon tumours were diagnosed in 22 patients (25.6\%), while $64(75.4 \%)$ patients had a left colon tumour. Out of 22 right colon tumours, eight (36.3\%) were located in the cecum, nine (41\%) were in the ascending colon, and five $(22.7 \%)$ were in the transverse colon. There were 17 (26.6\%) malignancies in the descending colon, 24 (37.5\%) malignancies in the sigmoid colon, and 23 (35.9\%) malignancies in the rectum. Patients were followed up with a CT every six months for three years and then annually, with a maximum follow-up period of 72 months. The mean follow-up time was 38.5 months.

A total of 481 metastases were counted. The mean size of liver metastases was $28.6 \pm 14.1 \mathrm{~mm}$ (range; 11.5 $68.4 \mathrm{~mm}$ ). Fifty-five liver (11.6\%) metastases were diagnosed synchronously with the primary colorectal cancer at diagnosis. Out of 481 metastases, 319 (66.3\%) metasta-

Table 1. The number of metastases in the study population according to the hepatic lobar distribution and primary tumour location

\begin{tabular}{|l|c|c|c|c|c|} 
& Right hemiliver & Left hemiliver & Total & R:L & $p$ \\
\hline Right colon & 82 & 53 & 135 & $1.55: 1$ & 0.106 \\
\hline Left colon & 237 & 109 & 346 & $2.17: 1$ \\
\hline Total & 319 & 162 & 481 & $1.97: 1$ & \\
\hline
\end{tabular}

Table 2. Segmental distribution of hepatic metastases

\begin{tabular}{|l|c|c|c|}
\hline Liver involvement & Right colon, $n(\%)$ & Left colon, $n(\%)$ & Total, $n(\%)$ \\
\hline Segment 8 & $23(26.7)$ & $63(73.3)$ & $86(17.9)$ \\
\hline Segment 7 & $17(30.9)$ & $38(69.1)$ & $55(11.4)$ \\
\hline Segment 6 & $28(24.6)$ & $86(75.4)$ & $114(23.7)$ \\
\hline Segment 5 & $14(21.9)$ & $50(78.1)$ & $64(13.3)$ \\
\hline Segment 4a & $13(40.6)$ & $19(59.4)$ & $32(6.7)$ \\
\hline Segment 4b & $10(25.0)$ & $30(75.0)$ & $40(8.3)$ \\
\hline Segment 3 & $15(48.4)$ & $16(51.6)$ & $31(6.4)$ \\
\hline Segment 2 & $13(26.5)$ & $36(73.5)$ & $49(10.2)$ \\
\hline Segment 1 & $2(20.0)$ & $8(80.0)$ & $10(2.1)$ \\
\hline
\end{tabular}


ses were located in the right hemiliver. No significant difference was observed according to the lobar distribution of hepatic metastases between right and left colon tumour groups $(p=0.106)$. The ratio of hepatic metastases according to the involvement of the right and left hemilivers was $1.55: 1$ for right colon tumours and $2.17: 1$ for left colon tumours. Table 1 shows the number of metastases located at the right and left hemilivers in the study population.

A subgroup analysis was performed with unilobar metastatic patients $(n=43)$ to show the effect of portal streamline flow that might be applied for early disease. In the subgroup analysis, out of 43 patients, 31 had a leftsided tumour. However, distribution of lobar hepatic metastases did not reach a statistically significant difference when comparing left colon tumours to right colon tumours $(p=0.325)$.

In the segmental distribution of metastases, metastases were observed at least in the caudate lobe (2\%). The most common site of metastases was segment VI (23\%), followed by segments VIII (18\%) and V (13\%). There was no significant difference in terms of segmental involvement between right and left colon tumour groups $(p>0.05)$. Table 2 represents the segmental distribution of metastases according to the location of the tumour.

\section{Discussion}

Streamline hypothesis refers to the unequal mixture of the SMV and IMV in the portal venous system, which influences non-proportional anatomical distribution of blood through the right and left hemilivers [7-10]. Experimental studies have indicated that blood in the SMV mostly flows into the right hemiliver, while the IMV disperses throughout the liver homogenously. Based on this hypothesis, studies have investigated the relation between the site of the colonic tumour and its hepatic metastases.

The current study showed no significant difference in the distribution of lobar hepatic metastasis according to the primary location of the colorectal tumour. The metastatic deposits derived from right and left colon tumours tend to metastasise to the right hemiliver with a ratio of $1.55: 1$ and $2.17: 1$, which is proportional to the liver's volume. In concordance with our results, an autopsy study conducted by Schulz et al. [11] showed homogenous distribution of hepatic metastases, and they did not observe a relationship between the primary tumour location and hepatic metastasis. They assumed that different metastatic pathways might be involved in colorectal carcinoma especially in an advanced stage of disease rather than the portal way (transarterial spread from other sources), and they added that the streamline hypothesis might be applied for the early stage of disease. In the current study, to avoid the effect of secondary metastatic routes, the patients with extrahepatic distant metastases such as lung, bone, and peritoneal were excluded. Also, to investigate the streamline flow in the portal vein, the lowermid rectal tumours were not included in the study because lower-mid rectal tumours have systemic caval venous drainage. The current study also included bilobar metastatic patients in whom the metastasis might be spread via intralobar branches of the hepatic vein, perineural space, or intrahepatic biliary tracts. However, to verify the streamline hypothesis, we accomplished a subgroup analyses with the patients who had unilobar metastases. Unfortunately, the distribution of hepatic metastases did not show any significant difference according to the location of the primary colon tumour.

Metastatic spread is a multistep process and is not randomly organised. According to Stephen Paget's seed and soil hypothesis [12], the metastatic process is composed of multiple steps referred to as "invasion-metastasis cascade". Initial steps are the outgrowth of tumour cells (seeds) into the extracellular matrix and then invasion into the host's systemic vasculature. After intravasation, the tumour cells have to survive in the blood or lymphatic circulation. If they survive, they stop at the host (soil) organ and start to colonise [13-15]. Under these circumstances, as stated in the seed and soil hypothesis, the intravasation of tumour cells through the systemic blood circulation occurs either via the venous/ capillary system or via the lymphatic system. Both arterial and venous pathways might be involved, even in the early stage of the metastatic disease. This might explain why the result of the present study is inconsistent with the streamline hypothesis even in the early stage of the disease.

In addition, several investigators performed conflicting results in the literature [7,16-18]. In a study by Rhu et al., they claimed that streamline flow can induce the lobar distribution of hepatic metastases. They observed the right-to-left ratio of liver metastases in patients with right colon cancer as $2.20: 1$, with the ratio in left colon patients as $1.39: 1$ [16]. Similarly, a study conducted by Shirai et al. showed the predominance of right hemiliver involvement from right-sided colon cancers. They concluded that metastases from rightsided colon cancers tend to involve the right hemiliver, while metastases of left colon cancers are spread homogenously in the liver [17]. These studies confirmed the effect of the streamline hypothesis on the hepatic metastases, including only the resected patients who had undergone surgery. In these circumstances, the small metastatic tumour deposits that can be detected only via CT or other radiologic investigations were too small to be realised in surgery and were not analysed. This might be the cause of incompatibility of the current study with these previous studies. On the other hand, the study population of Rhu et al. involved 28 patients with simultaneous lung metastases [16]. In this situation, the secondary metastatic routes, beyond the portal-venous way, might have become involved, altering the results. In line with the current study, Wigmore et al. did not observe any difference in the lobar distribution of hepatic metastases of colon cancer according to the primary location of the tumour [7]. In their study, they indicated the homogenous distribution of hepatic metastases from both right- and leftsided tumours with a ratio of $2.1: 1$ and $2.02: 1$, respectively. The right hemiliver is larger than the left hemiliver, and the 
ratio of the right liver's volume to left lobe's volume is $2: 1$ $[18,19]$. Considering the ratio of the hemiliver volumes as $2: 1$, the ratio of metastases of colorectal tumours in the liver relative to the lobes should be $2: 1$. In the current study, the overall ratio of involvement of hepatic metastases without categorising according to the location of the primary tumour is $1.97: 1$, which is compatible with the above statement. But the ratio of hepatic involvement did not show any significant difference when the tumours were classified according to their location.

Nonetheless, this study has several limitations. The small sample size of study population and the study's retrospective design are the primary limitations. Secondly, the involvement of other metastatic routes beyond the portal vein in the follow-up patients with bilobar metastases might have influenced the results. To eliminate this unfavourable effect, a subgroup analysis was performed. However, the subgroup analysis was accomplished with 43 patients, among whom were only a limited number of right-sided colon cancer patients. This might have limited the accuracy of the current study result. With a larger prospective study cohort and with the selection of early-stage patients, the validity of the streamline hypothesis should be tested further. The other limitation is that $15 \%$ of the study population with hepatic metastases were not histopathologically proven. However, all the metastatic lesions included in the study incurred size increases in the follow-up studies.

\section{Conclusions}

Our data illustrated that there is no relationship between the distribution of hepatic metastases and colorectal tumour location with regard to dynamic alterations of the portal vein. We think that the potential location of liver metastasis according to the site of the colorectal tumour cannot be predicted by attributing the streamline phenomena and other metastatic pathways (based on the seed and soil hypothesis) to have an effect on this relation. Further prospective experimental studies are required to reach a valid conclusion.

\section{Conflict of interest}

The authors report no conflict of interest.

\section{References}

1. Wang Z, Wang X, Zhang Z, et al. Association between primary tumor location and prognostic survival in synchronous colorectal liver metastases after surgical treatment: a retrospective analysis of SEER data. J Cancer 2019; 10: 1593-1600.

2. Byun JH, Ahn JB, Kim SY, et al. The impact of primary tumor location in patients with metastatic colorectal cancer: a Korean Cancer Study Group CO12-04 study. Korean J Intern Med 2019; 34: 165-177.

3. Baran B, Mert Ozupek N, Yerli Tetik N, et al. Difference between left-sided and right-sided colorectal cancer: a focused review of literature. Gastroenterology Res 2018; 11: 264-273.

4. Mik M, Berut M, Dziki L, et al. Right- and left-sided colon cancerclinical and pathological differences of the disease entity in one organ. Arch Med Sci 2017; 13: 157-162.

5. Brenner H, Kloor M, Pox CP. Colorectal cancer. Lancet 2014; 383 : 1490-1502.

6. Valderrama-Treviño AI, Barrera-Mera B, Ceballos-Villalva JC, et al. Hepatic metastasis from colorectal cancer. Euroasian J Hepatogastroenterol 2017; 7 : 166-175.

7. Wigmore SJ, Madhavan K, Redhead DN, et al. Distribution of colorectal liver metastases in patients referred for hepatic resection. Cancer 2000; 89: 285-287.

8. Copher GH, Dick BM. "Streamline" phenomena in the portal vein and selective distribution of portal blood in the liver. Arch Surg 1928; 17: 408-419.

9. Moore GE, Bridenbaugh RB. Roentgen demonstration of the venous circulation in the liver; portal venography. Radiology 1951; 57: 685-690.

10. Gates GF, Dore EK. Streamline flow in the human portal vein. J Nucl Med 1973; 14: 79-83.

11. Schulz W, Hagen C, Hort W. The distribution of liver metastases from colonic cancer. A quantitative postmortem study. Virchows Arch A Pathol Anat Histopathol 1985; 406: 279-284

12. Paget $\mathrm{S}$. The distribution of secondary growths in cancer of the breast. Lancet 1889; 133: 571-573.

13. Faraji F, Eissenberg JC. Seed and soil: a conceptual framework of metastasis for clinicians. Mo Med 2013; 110: 302-308.

14. Akhtar M, Haider A, Rashid S, et al. Paget's "Seed and soil" theory of cancer metastasis: an idea whose time has come. Adv Anat Pathol 2019; 26: 69-74.

15. Seretis F, Seretis C, Youssef H, et al. Colorectal cancer: seed and soil hypothesis revisited. Anticancer Res 2014; 34: 2087-2094.

16. Rhu J, Heo JS, Choi SH, et al. Streamline flow of the portal vein affects the lobar distribution of colorectal liver metastases and has a clinical impact on survival. Ann Surg Treat Res 2017; 92: 348-354.

17. Shirai Y, Wakai T, Ohtani T, et al. Colorectal carcinoma metastases to the liver. Does primary tumor location affect its lobar distribution? Cancer 1996; 77: 2213-2216.

18. Konopke R, Distler M, Ludwig S, et al. Location of liver metastases reflects the site of the primary colorectal carcinoma. Scand J Gastroenterol 2008; 43: 192-195.

19. Strohmeyer T, Schultz W. The distribution of metastases of different primary tumors in the liver. Liver 1986; 6: 184-187. 